\title{
Integrable systems on the sphere associated with genus three algebraic curves
}

\author{
A. V. Tsiganov, V. A. Khudobakhshov \\ St.Petersburg State University, St.Petersburg, Russia \\ e-mail: andrey.tsiganov@gmail.com,vitaly.khudobakhshov@gmail.com
}

\begin{abstract}
New variables of separation for few integrable systems on the two-dimensional sphere with higher order integrals of motion are considered in detail. We explicitly describe canonical transformations of initial physical variables to the variables of separation and vice versa, calculate the corresponding quadratures and discuss some possible integrable deformations of initial systems .
\end{abstract}

\section{Introduction}

A fundamental requirement for new developments in mechanics is to unravel the geometry that underlies different dynamical systems, especially mechanical systems. There are several reasons why this geometrical understanding is fundamental. First, it is a key tool for reduction by symmetries and for the geometric characterization of the integrability and stability theories. Second, the effective use of numerical techniques is often based on the comprehension of the fundamental structures appearing in the dynamics of mechanical and control systems. In fact, geometric analysis of such systems reveals what they have in common and indicates the most suitable strategy to obtain and to analyze their solutions.

Already in 19th century Euler and Lagrange established a mathematically satisfactory foundation of Newtonian mechanics. In 10. Jacobi united their ideas with the Hamilton optic theory and with the Abel geometric methods at a new Hamilton-Jacobi formalism. The Hamilton-Jacobi formalism was a crucial step towards Liouvilles classical definition of the notion of integrability [15] based on the notion of first integrals of motion.

The Liouville definition of integrable Hamiltonian systems naturally covered many classical examples. Among them are the Kepler motion solved by Newton, harmonic oscillators solvable by trigonometric functions, the Euler and Lagrange spinning tops and the Jacobi example of geodesic motion on an ellipsoid solvable by elliptic functions [2, the Neumann system on the sphere [16] and Kowalevski top [13] solved terms of hyperelliptic functions etc. In novel times much attention owing to the another discovery of the vast class of integrable soliton nonlinear partial differential equation, that admits this type of integrability when dynamics is restricted to finite dimensional Liouville tori and the system appeared to be completely integrable in the Liouville-Arnold sense. They all are more or less connected with the hyperelliptic curves and with the hyperelliptic functions [1, 10, 17. Below we show that foregoing development of the theory detected a number of cases when associated algebraic curve is non hyperelliptic and and its genus exceeds the number degrees of freedom [24, 26, 27.

Bi-Hamiltonian structures can be seen as a dual formulation of integrability and separability, in the sense that they substitute a hierarchy of compatible Poisson structures to the hierarchy of functions in involution, which may be treated either as integrals of motion or as variables of separation for some dynamical system [20]. The Eisenhart-Benenti theory was embedded into the bi-Hamiltonian set-up using the lifting of the conformal Killing tensor that lies at the heart of Benenti's construction, which may be realized as a computer algorithm [9]. The concept of natural Poisson bivectors allows us to generalize this construction and to study systems with quadratic and higher order integrals of motion in framework of a single theory [20, 23, 24]. 
The aim of this note is to discuss separation of variables for integrable natural systems on the two-dimensional unit sphere $\mathbb{S}^{2}$ from $[21,22,23,24,26]$. In the above mentioned previous papers we focused our attention on the bi-Hamiltonian calculations of the variables of separation starting from the given integrals of motion. This note is devoted to construction of the initial physical variables in terms of variables of separation, to calculation of the corresponding quadratures and to discussion of the possible integrable "gyroscopic" deformations of these systems associated with genus three algebraic curves.

In order to describe integrable systems on the sphere we will use the angular momentum vector $J=\left(J_{1}, J_{2}, J_{3}\right)$ and the Poisson vector $x=\left(x_{1}, x_{2}, x_{3}\right)$ in a moving frame of coordinates attached to the principal axes of inertia. The Poisson brackets between these variables

$$
\left\{J_{i}, J_{j}\right\}=\varepsilon_{i j k} J_{k}, \quad\left\{J_{i}, x_{j}\right\}=\varepsilon_{i j k} x_{k}, \quad\left\{x_{i}, x_{j}\right\}=0,
$$

may be associated to the Lie-Poisson brackets on the algebra $e^{*}(3)$. Using the Hamilton function $H$ and the Lie-Poisson bracket $\{.,$.$\} (1.1) on the Euclidean algebra e^{*}(3)$ the customary Euler-Poisson equations may be rewritten in the Hamiltonian form

$$
\dot{J}_{i}=\left\{J_{i}, H\right\}, \quad \dot{x}_{i}=\left\{x_{i}, H\right\}
$$

Remind, that the Lie-Poisson dynamics on $e^{*}(3)$ can be interpreted as resulting from reduction by the symmetry Euclidean group $E(3)$ of the full dynamics on the twelve-dimensional phase space $T^{*} E(3)$ [2]. There are two Casimir elements

$$
C_{1}=|x|^{2} \equiv \sum_{k=1}^{3} x_{k}^{2}, \quad C_{2}=\langle x, J\rangle \equiv \sum_{k=1}^{3} x_{k} J_{k}
$$

where $\langle.,$.$\rangle means inner product. Using canonical transformations x \rightarrow \alpha x$ we will always put $C_{1}=1$ without loss of generality.

If the square integral of motion $C_{2}=\langle x, J\rangle$ is equal to zero, rigid body dynamics may be restricted on the unit sphere $\mathbb{S}^{2}$ and we can use standard spherical coordinate system on its cotangent bundle $T^{*} \mathbb{S}^{2}$

$$
\begin{array}{llrl}
x_{1}=\sin \phi \sin \theta, & x_{2}=\cos \phi \sin \theta, & x_{3}=\cos \theta, \\
J_{1}=\frac{\sin \phi \cos \theta}{\sin \theta} p_{\phi}-\cos \phi p_{\theta}, & J_{2}=\frac{\cos \phi \cos \theta}{\sin \theta} p_{\phi}+\sin \phi p_{\theta}, & J_{3}=-p_{\phi} .
\end{array}
$$

We use these variables in order to determine canonical variables of separation on $T^{*} \mathbb{S}^{2}$.

As usual all the results are presented up to the linear canonical transformations, which consist of rotations

$$
x \rightarrow \alpha U x, \quad J \rightarrow U J,
$$

where $\alpha$ is an arbitrary parameter and $U$ is an orthogonal constant matrix, and shifts

$$
x \rightarrow x, \quad J \rightarrow J+S x,
$$

where $S$ is an arbitrary $3 \times 3$ skew-symmetric constant matrix.

Of course, any canonical transformation of the spherical variables (1.4) yields automorphism of $e^{*}(3)$ too. For instance, trivial canonical transformation

$$
p_{\theta} \rightarrow p_{\theta}+f(\theta)
$$

gives rise to "generalized" shift depending on arbitrary function $f\left(x_{3}\right)$ :

$$
J_{1} \rightarrow J_{1}-\frac{x_{2} f\left(x_{3}\right)}{\sqrt{x_{1}^{2}+x_{2}^{2}}}, \quad J_{2} \rightarrow J_{2}+\frac{x_{1} f\left(x_{3}\right)}{\sqrt{x_{1}^{2}+x_{2}^{2}}}
$$

This and more complicated canonical transformations of $e^{*}(3)$ are discussed in [2, 11]. 


\section{Kowalevski top and Chaplygin system}

Following to [21, 22, 24, we determine canonical coordinates $q_{1,2}$ on $T^{*} \mathbb{S}^{2}$ as roots of the following polynomial

$$
\begin{aligned}
B(\lambda) & =\left(\lambda-q_{1}\right)\left(\lambda-q_{2}\right)=\lambda^{2}-\frac{p_{\theta}^{2} \sin ^{2} \theta+p_{\phi}^{2} \cos ^{2} \theta}{\sin ^{\alpha} \theta \cos ^{2} \theta} \lambda-a^{2}-b^{2} \\
& -\frac{(a \cos \alpha \phi-b \sin \alpha \phi)\left(p_{\theta}^{2} \sin ^{2} \theta+p_{\phi}^{2} \cos ^{2} \theta\right)}{\sin ^{\alpha} \theta \cos ^{2} \theta}-\frac{2 \sin \theta(a \sin \alpha \phi+b \cos \alpha \phi) p_{\phi} p_{\theta}}{\sin ^{\alpha} \theta \cos ^{2} \theta} .
\end{aligned}
$$

Then we can introduce auxiliary polynomial

$$
A(\lambda)=\frac{\sin \theta p_{\theta}}{\alpha \cos \theta} \lambda+\frac{a \sin \alpha \phi+b \cos \alpha \phi}{\alpha} p_{\phi}-\frac{\sin \theta(a \cos \alpha \phi-b \sin \alpha \phi)}{\alpha \cos \theta} p_{\theta},
$$

such that

$$
\{B(\lambda), A(\mu)\}=\frac{1}{\lambda-\mu}\left(\left(\mu^{2}-a^{2}-b^{2}\right) B(\lambda)-\left(\lambda^{2}-a^{2}-b^{2}\right) B(\mu)\right), \quad\{A(\lambda), A(\mu)\}=0 .
$$

It entails that

$$
p_{j}=-\frac{1}{u_{j}^{2}-a^{2}-b^{2}} A\left(\lambda=q_{j}\right), \quad j=1,2,
$$

are canonically conjugated momenta on $T^{*} \mathbb{S}^{2}$ with the standard Poisson brackets

$$
\left\{q_{i}, p_{j}\right\}=\delta_{i j}, \quad\left\{q_{1}, q_{2}\right\}=\left\{p_{1}, p_{2}\right\}=0 .
$$

Below we prove that at $\alpha=1,2$ this variables are variables of separation for the Kowalevski top and Chaplygin system, respectively.

\subsection{Kowalevski top}

Let us consider Kowalevski top defined by the following integrals of motion

$$
\begin{aligned}
& H_{1}=J_{1}^{2}+J_{2}^{2}+2 J_{3}^{2}+2 b x_{1} \\
& H_{2}=\left(J_{1}^{2}+J_{2}^{2}\right)^{2}-4 b\left(x_{1}\left(J_{1}^{2}-J_{2}^{2}\right)+2 x_{2} J_{1} J_{2}\right)-4 b^{2} x_{3}^{2} .
\end{aligned}
$$

In original Kowalevski work 13 the first step in the separation of variables method is the complexification: she introduces

$$
\mathrm{z}_{1}=J_{1}+i J_{2}, \quad \mathrm{z}_{2}=J_{1}-i J_{2}
$$

as independent complex variables. Next she makes her famous change of variables

$$
s_{1,2}=\frac{R\left(\mathrm{z}_{1}, \mathrm{z}_{2}\right) \pm \sqrt{R\left(\mathrm{z}_{1}, \mathrm{z}_{1}\right) R\left(\mathrm{z}_{2}, \mathrm{z}_{2}\right)}}{2\left(\mathrm{z}_{1}-\mathrm{z}_{2}\right)^{2}} .
$$

The fourth degree polynomials $R\left(\mathrm{z}_{i}, \mathrm{z}_{k}\right)$ may be found in [13, 2]. It brings the system (1.2) to the form

$$
(-1)^{k}\left(s_{1}-s_{2}\right) \dot{s}_{k}=\sqrt{P\left(s_{k}\right)}, \quad k=1,2,
$$

where

$$
P(s)=4\left(\left(s-H_{1}\right)^{2}-\frac{H_{2}+4 b^{2} C_{1}}{4}\right)\left[s\left(\left(s-H_{1}\right)^{2}+b^{2} C_{1}-\frac{H_{2}+4 a^{2} C_{1}}{4}\right)+b^{2} C_{2}\right] .
$$


Consequently, initial equations of motion can be written as hyperelliptic quadratures

$$
\begin{aligned}
& \frac{\dot{s}_{1}}{\sqrt{P\left(s_{1}\right)}}+\frac{\dot{s}_{2}}{\sqrt{P\left(s_{2}\right)}}=0, \\
& \frac{s_{1} \dot{s}_{1}}{\sqrt{P\left(s_{1}\right)}}+\frac{s_{2} \dot{s}_{2}}{\sqrt{P\left(s_{2}\right)}}=\mathrm{i},
\end{aligned}
$$

where we can substitute the conjugated momenta $p_{s_{k}}$ instead of $\sqrt{P\left(s_{k}\right)}$ in order to get standard Abel-Jacobi form. So, the problem can be integrated in term of genus two hyperelliptic functions of time. Finally, we have to substitute these functions of time $s_{k}(t)$ and $p_{s_{k}}(t)$ into the initial variables $x, J$, the corresponding expressions may be found in [13, 12].

Discussion of the another variables of separation for some particular subcases in the Kowalevski dynamic may be found in [2]. As usual different variables of separation are related with the distinct integrable deformations of the initial integrals of motion.

\subsubsection{New real variables of separation at $C_{2}=0$}

According to [24], at $\alpha=1$ coordinates $q_{1,2}$ (2.1) are variables of separation associated with the Hamilton function

$$
H=J_{1}^{2}+J_{2}^{2}+2 J_{3}^{2}++2 a x_{2}+2 b x_{1},
$$

which may be reduced to the initial Hamiltonian $H_{1}$ using rotations (1.5) around the third axis [11, so we can put $a=0$ in (2.1) without loss of generality.

Coordinates $q_{1,2}$ (2.1) at $\alpha=1$ and $a=0$ are defined by

$$
B(\lambda)=\left(\lambda-q_{1}\right)\left(\lambda-q_{2}\right)=\lambda^{2}+\frac{\sqrt{x_{1}^{2}+x_{2}^{2}}\left(J_{1}^{2}+J_{2}^{2}\right)}{x_{3}^{2}}-\frac{b\left(x_{1}\left(J_{1}^{2}-J_{2}^{2}\right)+2 x_{2} J_{1} J_{2}\right)}{x_{3}^{2}}-b^{2} .
$$

The conjugated momenta $p_{1,2}$ are equal to

$$
p_{k}=-\frac{A\left(\lambda=q_{k}\right)}{q_{k}^{2}-b^{2}}, \quad A(\lambda)=\frac{x_{1} J_{2}-x_{2} J_{1}}{x_{3}} \lambda+\frac{b \sqrt{x_{1}^{2}+x_{2}^{2}} J_{2}}{x_{3}} .
$$

This variables $q_{1,2}$ are differed on a constant terms $\pm b$ from variables introduced in 22. Inverse transformation reads as

$$
\begin{aligned}
& x_{1}=\frac{b^{2}-q_{1} q_{2}}{b\left(q_{1}-q_{2}\right)^{2}}\left(\left(b^{2}-q_{1}^{2}\right) p_{1}^{2}+\left(b^{2}-q_{2}^{2}\right) p_{2}^{2}\right)-\frac{2\left(b^{2}-q_{1}^{2}\right)\left(b^{2}-q_{2}^{2}\right)}{b\left(q_{1}-q_{2}\right)^{2}} p_{1} p_{2}, \\
& x_{2}=-\frac{\sqrt{\left(q_{1}^{2}-b^{2}\right)\left(b^{2}-q_{2}^{2}\right)}}{b\left(q_{1}-q_{2}\right)^{2}}\left(b^{2}\left(p_{1}-p_{2}\right)^{2}-\left(p_{1} q_{1}-p_{2} q_{2}\right)^{2}\right) \\
& x_{3}=\sqrt{1-\frac{\left(b^{2}-q_{1}^{2}\right)^{2} p_{1}^{4}+\left(b^{2}-q_{2}^{2}\right)^{2} p_{2}^{4}}{\left(q_{1}-q_{2}\right)^{2}}+\frac{2\left(b^{2}-q_{1}^{2}\right)\left(b^{2}-q_{2}^{2}\right) p_{1}^{2} p_{2}^{2}}{\left(q_{1}-q_{2}\right)^{2}}} \\
& J_{1}=\frac{\sqrt{\left(q_{1}^{2}-b^{2}\right)\left(b^{2}-q_{2}^{2}\right)}\left(p_{1} q_{1}-p_{2} q_{2}\right)}{\left(b^{2}-q_{1}^{2}\right) p_{1}^{2}-\left(b^{2}-q_{2}^{2}\right) p_{2}^{2}} \frac{x_{3}}{b} \\
& J_{2}=-\frac{q_{2}\left(b^{2}-q_{1}^{2}\right) p_{1}-q_{1}\left(b^{2}-q_{2}^{2}\right) p_{2}}{\left(b^{2}-q_{1}^{2}\right) p_{1}^{2}-\left(b^{2}-q_{2}^{2}\right) p_{2}^{2}} \frac{x_{3}}{b} \\
& J_{3}=-\sqrt{\left(q_{1}^{2}-b^{2}\right)\left(b^{2}-q_{2}^{2}\right)} \frac{p_{1}-p_{2}}{q_{1}-q_{2}} .
\end{aligned}
$$

Coordinates of separation take values only in the following intervals

$$
q_{1}>b>q_{2},
$$


similar to the standard elliptic coordinates on the sphere [2].

In this variables integrals of motion $H_{1,2}(2.3)$ look like

$$
\begin{aligned}
H_{1} & =\frac{\left(b^{2}-q_{1}^{2}\right)^{2} p_{1}^{4}-\left(b^{2}-q_{2}^{2}\right)^{2} p_{2}^{4}-\left(q_{1}^{2}-q_{2}^{2}\right)}{\left(b^{2}-q_{1}^{2}\right) p_{1}^{2}-\left(b^{2}-q_{2}^{2}\right) p_{2}^{2}} \\
H_{2} & =\frac{\left(\left(b^{2}-q_{1}^{2}\right) p_{1}^{2}-\left(b^{2}-q_{2}^{2}\right) p_{2}^{2}+q_{1}+q_{2}\right)\left(\left(b^{2}-q_{1}^{2}\right) p_{1}^{2}-\left(b^{2}-q_{2}^{2}\right) p_{2}^{2}-q_{1}-q_{2}\right)}{\left(b^{2}-q_{1}^{2}\right) p_{1}^{2}-\left(b^{2}-q_{2}^{2}\right) p_{2}^{2}} \\
& \times\left(\left(b^{2}-q_{1}^{2}\right) p_{1}^{2}-\left(b^{2}-q_{2}^{2}\right) p_{2}^{2}+q_{1}-q_{2}\right)\left(\left(b^{2}-q_{1}^{2}\right) p_{1}^{2}-\left(b^{2}-q_{2}^{2}\right) p_{2}^{2}-q_{1}+q_{2}\right) .
\end{aligned}
$$

It is easy to see that integrals of motion and variables of separation are related via the following separated relations

$$
\Phi=\left(2\left(q^{2}-b^{2}\right) p^{2}+H_{1}+\sqrt{H_{2}}\right)\left(2\left(q^{2}-b^{2}\right) p^{2}+H_{1}-\sqrt{H_{2}}\right)-4 q^{2}=0,
$$

at $q=q_{1,2}$ and $p=p_{1,2}$. Equation $\Phi(q, p)=0$ defines genus three hyperelliptic curve with the following base of the holomorphic differentials

$$
\begin{aligned}
& \Omega_{1}=\frac{d q}{p\left(b^{2}-q^{2}\right)\left(H_{1}-2\left(b^{2}-q^{2}\right) p^{2}\right)}, \quad \Omega_{2}=\frac{q d q}{p\left(b^{2}-q^{2}\right)\left(H_{1}-2\left(b^{2}-q^{2}\right) p^{2}\right)} \\
& \Omega_{3}=\frac{p d q}{H_{1}-2\left(b^{2}-q^{2}\right) p^{2}}, .
\end{aligned}
$$

In fact equation (2.6) is invariant with respect to involution $(q, p) \rightarrow(-q, p)$. Factorization with respect to this involution give rise to elliptic curve.

In variables of separation equations of motion (1.2) have the following form

$$
\begin{gathered}
\frac{\dot{q}_{1}}{p_{1}\left(b^{2}-q_{1}^{2}\right)\left(H_{1}-2\left(b^{2}-q_{1}^{2}\right) p_{1}^{2}\right)}+\frac{\dot{q}_{2}}{p_{2}\left(b^{2}-q_{2}^{2}\right)\left(H_{1}-2\left(b^{2}-q_{2}^{2}\right) p_{2}^{2}\right)}=0, \\
\frac{\dot{q}_{1}}{H_{1}-2\left(b^{2}-q_{1}^{2}\right) p_{1}^{2}}+\frac{\dot{q}_{2}}{H_{1}-2\left(b^{2}-q_{2}^{2}\right) p_{2}^{2}}=-2 .
\end{gathered}
$$

The above quadratures in the integral form

$$
\int_{q_{0}}^{q_{1}} \Omega_{1}+\int_{q_{0}}^{q_{2}} \Omega_{1}=\beta_{1}, \quad \int_{q_{0}}^{q_{1}} \Omega_{3}+\int_{q_{0}}^{q_{2}} \Omega_{3}=-2 t+\beta_{2}
$$

represent the Abel-Jacobi map associated to the genus three hyperelliptic curve defined by $\Phi(q, p)=0$. In particular it means that instead of $p$ in $\Omega_{1,3}$ (2.7) we have to substitute function on $q$ obtained from the separated relation (2.6).

In order to give explicit theta-functions solution one can apply some remarkable relations between roots of certain functions on symmetric products of such curves and quotients of theta-functions with half-integer characteristics, which are historically referred to as root function and are generalized socalled Wurzelfunktionen that were used by Jacobi for the case of ordinary hyperelliptic Jacobians 3, 6. For the case of odd order hyperelliptic curves such functions were obtained by Weierstrass 28 . Inverting the map (2.7) and substituting symmetric functions of $q_{1}, q_{2}, p_{1}, p_{2}$ into (2.5), one finally finds $x, J$ as functions of time.

\subsubsection{Deformations of the Kowalewski top}

According to 22, 24, using separated relations

$$
\Phi_{1}=\left(2\left(q^{2}-a^{2}\right) p^{2}+\widehat{H}_{1}+\sqrt{\widehat{H}_{2}}\right)\left(2\left(q^{2}-a^{2}\right) p^{2}+\widehat{H}_{1}-\sqrt{\widehat{H}_{2}}\right)-4 c u^{2}+4 d u+e\left(q^{2}-a^{2}\right) p=0,
$$


one gets Hamilton function of the generalized Kowalevski top

$$
\widehat{H}_{1}=\left(1-\frac{c-1}{x_{3}^{2}}\right)\left(J_{1}^{2}+J_{2}^{2}\right)+2 J_{3}^{2}+2 b x_{1}+\frac{d}{\sqrt{x_{1}^{2}+x_{2}^{2}}}+\frac{e\left(x_{2} J_{1}-x_{1} J_{2}\right)}{4 \sqrt{\left(x_{1}^{2}+x_{2}^{2}\right.} x_{3}} .
$$

Second integral of motion is equal to

$$
\begin{aligned}
\widehat{H}_{2} & =\frac{\left(x_{3}^{2}+c-1\right)^{2}}{x_{3}^{4}}\left(J_{1}^{2}+J_{2}^{2}\right)^{2}-\left(\frac{4\left(x_{1} J_{1}^{2}-x_{1} J_{2}^{2}+2 x_{2} J_{1} J_{2}\right)\left(x_{3}^{2}+c-1\right)}{x_{3}^{2}}+\frac{J_{2} \sqrt{x_{1}^{2}+x_{2}^{2}} e}{x_{3}}\right) a \\
& -4\left(x_{3}^{2}+c-1\right) a^{2}+\left(\frac{\left(2\left(J_{1}^{2}+J_{2}^{2}\right)\left(c-x_{1}^{2}-x_{2}^{2}\right)\right.}{\sqrt{x_{1}^{2}+x_{2}^{2}} x_{3}^{2}}+\frac{\left(x_{2} J_{1}-x_{1} J_{2}\right) e}{2\left(x_{1}^{2}+x_{2}^{2}\right) x_{3}}\right) d+\frac{d^{2}}{x_{1}^{2}+x_{2}^{2}} \\
& +\frac{\left(J_{1}^{2}+J_{2}^{2}\right)\left(x_{2} J_{1}-x_{1} J_{2}\right)\left(c-x_{1}^{2}-x_{2}^{2}\right) e}{2 \sqrt{x_{1}^{2}+x_{2}^{2}} x 3^{3}}+\frac{\left(x_{2} J_{1}-x_{1} J_{2}\right)^{2} e^{2}}{16\left(x_{1}^{2}+x_{2}^{2}\right) x_{3}^{2}}
\end{aligned}
$$

According to [11, 24] canonical transformation (1.8) reduces Hamilton function (2.9) to the natural form

$$
\widehat{H}_{1}=\left(1-\frac{c-1}{x_{3}^{2}}\right)\left(J_{1}^{2}+J_{2}^{2}\right)+2 J_{3}^{2}+2 a x_{1}+\frac{d}{\sqrt{x_{1}^{2}+x_{2}^{2}}}-\frac{e^{2}}{64\left(x_{3}^{2}+c-1\right)},
$$

at

$$
f\left(x_{3}\right)=\frac{e x_{3}}{8\left(x_{3}^{2}+c-1\right)} .
$$

At $c=1$ this system coincides with one of the deformations discussed in 29]. Below we will show only the final form (2.11) of the deformed Hamiltonians and will omit the intermediate form (2.9) for the brevity.

It is easy to calculate the corresponding equations of motion

$$
\begin{aligned}
\frac{\dot{q}_{1}}{\left(b^{2}-q_{1}^{2}\right)\left(8 \widehat{H}_{1} p_{1}+e-16 p_{1}^{3}\left(b^{2}-q_{1}^{2}\right)\right)}+\frac{\dot{q}_{2}}{p_{2}\left(b^{2}-q_{2}^{2}\right)\left(8 \widehat{H}_{1} p_{2}+e-16 p_{2}^{3}\left(b^{2}-q_{2}^{2}\right)\right)} & =0, \\
\frac{\dot{q}_{1}}{8 \widehat{H}_{1} p_{1}+e-16 p_{1}^{3}\left(b^{2}-q_{1}^{2}\right)}+\frac{\dot{q}_{2}}{8 \widehat{H}_{1} p_{2}+e-16 p_{2}^{3}\left(b^{2}-q_{2}^{2}\right)} & =-\frac{1}{4},
\end{aligned}
$$

and prove that the Abel-Jacobi map on genus three hyperelliptic curve has the same form (2.7)

$$
\int_{q_{0}}^{q_{1}} \Omega_{1}+\int_{q_{0}}^{q_{2}} \Omega_{1}=\beta_{1}, \quad \int_{q_{0}}^{q_{1}} \Omega_{3}+\int_{q_{0}}^{q_{2}} \Omega_{3}=-2 t+\beta_{2},
$$

where $p$ have to be solution of the separated relation (2.8) and

$$
\Omega_{1}=\frac{d q}{\left(b^{2}-q^{2}\right)\left(8 \widehat{H}_{1} p+e-16 p^{3}\left(b^{2}-q^{2}\right)\right)}, \quad \Omega_{3}=\frac{d q}{8 \widehat{H}_{1} p+e-16 p^{3}\left(b^{2}-q^{2}\right)} .
$$

\subsection{Chaplygin system}

Let us consider Chaplygin system defined by the following Hamilton function

$$
H_{1}=J_{1}^{2}+J_{2}^{2}+2 J_{3}^{2}-2 a\left(x_{1}^{2}-x_{2}^{2}\right)-2 b x_{1} x_{2}-\frac{c}{x_{3}^{2}} .
$$

At $c=0$ this system and the corresponding variables of separation have been investigated by Chaplygin 4]. Singular term has been added by Goryachev in 8 .

Using rotations (1.5) around the third axis [11] we can put $b=0$ without loss of generality. In this case second integral of motion is equal to

$$
H_{2}=\left(J_{1}^{2}+J_{2}^{2}-\frac{c}{x_{3}^{2}}\right)^{2}-4 a x_{3}^{2}\left(J_{1}^{2}-J_{2}^{2}\right)+4 a^{2} x_{3}^{4}
$$


According to [21, 24], coordinates $q_{1,2}$ (2.1) are variables of separation for this integrable system at $\alpha=2$ and $b=0$. In this case $q_{1,2}$ are roots of the following polynomial (2.1)

$$
B(\lambda)=\left(\lambda-q_{1}\right)\left(\lambda-q_{2}\right)=\lambda^{2}-\frac{J_{1}^{2}+J_{2}^{2}}{x_{3}^{2}} \lambda-\frac{2 a J_{2}^{2}}{x_{3}^{2}}+\frac{a\left(J_{1}^{2}+J_{2}^{2}\right)}{x_{3}^{2}}-a^{2},
$$

whereas momenta $p_{1,2}$ are values of the other auxiliary polynomial

$$
A(\lambda)=-\frac{x_{2} J_{1}-x_{1} J_{2}}{2 x_{3}} \lambda-\frac{a x_{1} x_{2} J_{3}}{x_{1}^{2}+x_{2}^{2}}-\frac{a\left(x_{1}^{2}-x_{2}^{2}\right)\left(x_{2} J_{1}-x_{1} J_{2}\right)}{2\left(x_{1}^{2}+x_{2}^{2}\right) x_{3}} .
$$

at $\lambda=q_{1,2}$ (2.2). Inverse transformation reads as

$$
\begin{aligned}
& x_{1}=\frac{\sqrt{2\left(q_{1}-a\right)\left(a-q_{2}\right)}\left(p_{1}\left(q_{1}+a\right)-p_{2}\left(q_{2}+a\right)\right)}{\sqrt{a}\left(q_{1}-q_{2}\right)}, \\
& x_{2}=\frac{\sqrt{2\left(q_{1}+a\right)\left(q_{2}+a\right)}\left(p_{1}\left(q_{1}-a\right)-p_{2}\left(q_{2}-a\right)\right)}{\sqrt{a}\left(q_{1}-q_{2}\right)}, \\
& x_{3}=\sqrt{1-4 \frac{\left(q_{1}^{2}-a^{2}\right) p_{1}^{2}-\left(q_{2}^{2}-a^{2}\right) p_{2}^{2}}{q_{1}-q_{2}}}, \\
& J_{1}=\sqrt{\frac{\left(a+q_{1}\right)\left(a+q_{2}\right)}{2 a}} x_{3}, \quad J_{2}=-\sqrt{\frac{\left(q_{1}-a\right)\left(a-q_{2}\right)}{2 a}} x_{3} \\
& J_{3}=-2 \sqrt{\left(q_{1}^{2}-a^{2}\right)\left(a^{2}-q_{2}^{2}\right)} \frac{p_{1}-p_{2}}{q_{1}-q_{2}}
\end{aligned}
$$

As usual coordinates of separation take values only in the following intervals

$$
q_{1}>a>q_{2} .
$$

This variables $q_{1,2}$ are related with variables of separation from [21] by the rule $q_{k} \rightarrow q_{k}+a$.

In variables of separation integrals of motion read as

$$
\begin{aligned}
H_{1} & =4\left(a^{2}-q_{1}^{2}\right) p_{1}^{2}+4\left(a^{2}-q_{2}^{2}\right) p_{2}^{2}+q_{1}+q_{2}-\frac{c}{4\left(a^{2}-q_{1}^{2}\right) p_{1}^{2}-4\left(a^{2}-q_{2}^{2}\right) p_{2}^{2}+q_{1}-q_{2}}, \\
H_{2} & =\left(4\left(a^{2}-q_{1}^{2}\right) p_{1}^{2}-4\left(a^{2}-q_{2}^{2}\right) p_{2}^{2}+q_{1}-q_{2}\right)^{2}+\frac{c^{2}\left(q_{1}-q_{2}\right)^{2}}{\left(4\left(a^{2}-q_{1}^{2}\right) p_{1}^{2}-4\left(a^{2}-q_{2}^{2}\right) p_{2}^{2}+q_{1}-q_{2}\right)^{2}} \\
& -2 c\left(q_{1}+q_{2}\right) .
\end{aligned}
$$

It is easy to see that integrals of motion and variables of separation are related via the following separated relations

$$
\Phi=\left(8\left(q^{2}-a^{2}\right) p^{2}-2 q+H_{1}-\sqrt{H_{2}}\right)\left(8\left(q^{2}-a^{2}\right) p^{2}-2 q+H_{1}+\sqrt{H_{2}}\right)-4 c q=0,
$$

at $q=q_{1,2}$ and $p=p_{1,2}$. Equation $\Phi(q, p)=0$ defines genus two algebraic curve with the following holomorphic differentials

$$
\Omega_{1}=\frac{d q}{p\left(a^{2}-q^{2}\right)\left(H_{1}-8\left(a^{2}-q^{2}\right) p^{2}-2 q\right)}, \quad \Omega_{2}=\frac{\left(4\left(a^{2}-q^{2}\right) p^{2}+q\right) d q}{p\left(a^{2}-q^{2}\right)\left(H_{1}-8\left(a^{2}-q^{2}\right) p^{2}-2 q\right)} .
$$


The corresponding quadratures look like

$$
\begin{aligned}
& \frac{\dot{q}_{1}}{p_{1}\left(a^{2}-q_{1}^{2}\right)\left(H_{1}-8\left(a^{2}-q_{1}^{2}\right) p_{1}^{2}-2 q_{1}\right)}+\frac{\dot{q}_{2}}{p_{2}\left(a^{2}-q_{2}^{2}\right)\left(H_{1}-8\left(a^{2}-q_{2}^{2}\right) p_{2}^{2}-2 q_{2}\right)}=0 \\
& \frac{\left(4\left(a^{2}-q_{1}^{2}\right) p_{1}^{2}+q_{1}\right) \dot{q}_{1}}{p_{1}\left(a^{2}-q_{1}^{2}\right)\left(H_{1}-8\left(a^{2}-q_{1}^{2}\right) p_{1}^{2}-2 q_{1}\right)}+\frac{\left(4\left(a^{2}-q_{2}^{2}\right) p_{2}^{2}+q_{2}\right) \dot{q}_{2}}{p_{2}\left(a^{2}-q_{2}^{2}\right)\left(H_{1}-8\left(a^{2}-q_{2}^{2}\right) p_{2}^{2}-2 q_{2}\right)}=8 .
\end{aligned}
$$

The Abel-Jacobi map on genus two hyperelliptic curve has the standard form

$$
\int_{q_{0}}^{q_{1}} \Omega_{1}+\int_{q_{0}}^{q_{2}} \Omega_{1}=\beta_{1}, \quad \int_{q_{0}}^{q_{1}} \Omega_{2}+\int_{q_{0}}^{q_{2}} \Omega_{2}=8 t+\beta_{2},
$$

where $p$ into $\Omega_{1,2}$ have to be solution of the separated relation (2.14).

\subsubsection{Deformations of the Chaplygin system}

According to [21, 24], if we substitute this variables of separation onto the following separated relations

$$
\Phi_{1}=\left(8\left(q^{2}-a^{2}\right) p^{2}-2 d q+\widehat{H}_{1}-\sqrt{\widehat{H}_{2}}\right)\left(8\left(q^{2}-a^{2}\right) p^{2}-2 d q+\widehat{H}_{1}+\sqrt{\widehat{H}_{2}}\right)-4 c q+e\left(q^{2}-a^{2}\right) p=0,
$$

one gets the Hamilton function of the generalized Chaplygin system

$$
\widehat{H}_{1}=\left(1-\frac{1-d}{x_{3}^{2}}\right)\left(J_{1}^{2}+J_{2}^{2}\right)+2 J_{3}^{2}-2 a\left(x_{1}^{2}-x_{2}^{2}\right)-2 b x_{1} x_{2}-\frac{c}{d-1+x_{3}^{2}}+\frac{\left(x_{2} J_{1}-x_{1} J_{2}\right) e}{8\left(d-x_{1}^{2}-x_{2}^{2}\right) x_{3}} .
$$

As for the Kowalevski top, using canonical transformation (1.8) at

$$
f\left(x_{3}\right)=\frac{e x_{3} \sqrt{1-x_{3}^{2}}}{16\left(d-1+x_{3}^{2}\right)^{2}}
$$

we can reduce Hamilton function (2.17) to the natural Hamiltonian

$$
\widehat{H}_{1}=\left(1-\frac{1-d}{x_{3}^{2}}\right)\left(J_{1}^{2}+J_{2}^{2}\right)+2 J_{3}^{2}-2 a\left(x_{1}^{2}-x_{2}^{2}\right)-2 b x_{1} x_{2}-\frac{c}{d-1+x_{3}^{2}}+\frac{e\left(x_{3}^{2}-1\right)}{256\left(d-1+x_{3}^{2}\right)^{3}} .
$$

At $d=1$ additional term is equal to $e\left(x_{3}^{-4}-x_{3}^{-6}\right)$ and this system coincides with one of the deformations considered in [29].

In this case we have genus three hyperelliptic curve with holomorphic differentials

$$
\begin{aligned}
& \Omega_{1}=\frac{d q}{\left(a^{2}-q^{2}\right)\left(e+32 p\left(\widehat{H}_{1}-8\left(a^{2}-q^{2}\right) p^{2}-2 d q\right)\right)}, \\
& \Omega_{2}=\frac{q d q}{\left(a^{2}-q^{2}\right)\left(e+32 p\left(\widehat{H}_{1}-8\left(a^{2}-q^{2}\right) p^{2}-2 d q\right)\right)}, \\
& \Omega_{3}=\frac{p^{2} d q}{e+32 p\left(\widehat{H}_{1}-8\left(a^{2}-q^{2}\right) p^{2}-2 d q\right)},
\end{aligned}
$$

and the corresponding quadratures involve all this differentials

$$
\int_{q_{0}}^{q_{1}} \Omega_{1}+\int_{q_{0}}^{q_{2}} \Omega_{1}=\beta_{1}, \quad \int_{q_{0}}^{q_{1}}\left(4 \Omega_{2}+d \Omega_{3}\right)+\int_{q_{0}}^{q_{2}}\left(4 \Omega_{2}+d \Omega_{3}\right)=-\frac{t}{4}+\beta_{2},
$$

in contrast with other integrable systems on genus three algebraic curves considered in this note. 


\section{Integrable systems associated with trigonal curves}

According to [26, 24, 27, we introduce other coordinates $q_{1,2}$ on $T^{8} \mathbb{S}^{2}$ defined as roots of the following polynomial

$$
B(\lambda)=\left(\lambda-q_{1}\right)\left(\lambda-q_{2}\right)=\lambda^{2}-\mathrm{i} \sqrt{F} \lambda+\Lambda, \quad \mathrm{i}=\sqrt{-1},
$$

with coefficients

$$
F=\left(g(\theta) p_{\theta}-\mathrm{i} h(\theta) p_{\phi}\right)^{2}, \quad \Lambda=\alpha \exp \left(\mathrm{i} \phi-\int \frac{h(\theta)}{g(\theta)} d \theta\right)
$$

depending on arbitrary functions $g(\theta)$ and $h(\theta)$. As usual conjugated momenta $p_{1,2}$ are equal to

$$
p_{k}=A\left(\lambda=q_{k}\right), \quad A(\lambda)=\mathrm{i} \int \frac{d \theta}{g(\theta)}-\frac{\mathrm{i} p_{\phi}}{\lambda} .
$$

It is easy to prove, that these polynomials satisfy to the following relations

$$
\{B(\lambda), A(\mu)\}=\frac{\lambda}{\mu-\lambda}\left(\frac{B(\lambda)}{\lambda}-\frac{B(\mu)}{\mu}\right), \quad\{A(\lambda), A(\mu)\}=\{B(\lambda), B(\mu)\}=0,
$$

which give rise to canonical Poisson brackets

$$
\left\{q_{i}, p_{j}\right\}=\delta_{i j}, \quad\left\{q_{1}, q_{2}\right\}=\left\{p_{1}, p_{2}\right\}=0 .
$$

Substituting variables

$$
x=a q_{k}^{-1}, \quad z=a_{0} p_{k}, \quad k=1,2, \quad a, a_{0} \in \mathbb{R},
$$

into the generic equation of the $(3,4)$ algebraic curve

$$
\Phi(z, x)=z^{3}+\left(a_{1} x+a_{2}\right) z^{2}+\left(H_{1} x^{2}+b_{1} x+b_{2}\right) z+x^{4}+H_{2} x^{3}+c_{1} x^{2}+c_{2} x+c_{3}=0,
$$

and solving the resulting equations with respect to $H_{1,2}$, one gets the following Hamilton function

$$
H_{1}=T+V+\left(\frac{c_{2}+\mathrm{i} a_{0} b_{1} w_{2}-a_{0}^{2} a_{1} w_{2}^{2}}{a_{0} a w_{2}} h+\frac{2 a_{0} a_{1} w_{2}-\mathrm{i} b_{1}}{a w_{2}}\right) \mathrm{i} p_{\phi}-\frac{g w_{2}\left(c_{2}+\mathrm{i} a_{0} b_{1} w_{2}-a_{0}^{2} a_{1} w_{2}^{2}\right)}{a a_{0}} p_{\theta},
$$

where geodesic Hamiltonian $T$ and potential $V$ are equal to

$$
\begin{aligned}
T & =\left(\frac{a_{0}^{2}\left(h^{2} w_{2}^{2}-3 h w_{2}+3\right)}{a^{2}}-\frac{\mathrm{i} a_{0} a_{2}\left(h w_{2}-1\right)^{2}}{a^{2} w_{2}}-\frac{b_{2} h\left(h w_{2}-1\right)}{a^{2} w_{2}}+\frac{\mathrm{i} c_{3} h^{2}}{a_{0} a^{2} w_{2}}\right) p_{\phi}^{2} \\
& +\frac{\mathrm{i} g}{a^{2} w_{2}}\left(\left(2 a_{0}^{2} w_{2}^{3}-2 \mathrm{i} a_{0} a_{2} w_{2}^{2}-2 b_{2} w 2+\frac{2 \mathrm{i} c_{3}}{a_{0}}\right) h-3 a_{0}^{2} w_{2}^{2}+2 \mathrm{i} a_{0} a_{2} w_{2}+b_{2}\right) p_{\phi} p_{\theta} \\
& +\frac{g^{2}\left(a_{0} b_{2} w_{2}+\mathrm{i} a_{0}^{2} a_{2} w_{2}^{2}-a_{0}^{3} w_{2}^{3}-\mathrm{i} c_{3}\right)}{a^{2} a_{0} w_{2}} p_{\theta}^{2} \\
V & =-\frac{\mathrm{i} a^{2} \mathrm{e}^{-\mathrm{i} \phi}}{\alpha a_{0} w_{1} w_{2}}+\frac{\left(a_{0} b_{2} w_{2}+\mathrm{i} a_{0}^{2} a_{2} w_{2}^{2}-a_{0}^{3} w_{2}^{3}-\mathrm{i} c_{3}\right) \alpha w_{1} \mathrm{e}^{\mathrm{i} \phi}}{a_{0} a^{2} w_{2}}+\frac{\mathrm{i} c_{1}}{a_{0} w_{2}} .
\end{aligned}
$$

Here

$$
w_{1}=\exp \left(-\int \frac{h(\theta)}{g(\theta)} d \theta\right), \quad w_{2}=\int \frac{d \theta}{g(\theta)} .
$$

Second integral of motion $\mathrm{H}_{2}$ is a cubic polynomial in momenta $p_{\phi}$ and $p_{\theta}$.

The resulting Hamiltonian $H_{1}$ (3.7) has the natural form, if and only if

$$
2 a_{0} a_{1} w_{2}-\mathrm{i} b_{1}=0, \quad c_{2}+\mathrm{i} a_{0} b_{1} w_{2}-a_{0}^{2} a_{1} w_{2}^{2}=0 .
$$


So, because $w_{2} \neq 0$, we have to put

$$
a_{1}=b_{1}=c_{2}=0 .
$$

If we want to obtain diagonal metric, then we have to solve integral equation

$$
2 h\left(a_{0}^{3} w_{2}^{3}-\mathrm{i} a_{0}^{2} a_{2} w_{2}^{2}-a_{0} b_{2} w_{2}+\mathrm{i} c_{3}\right)-3 a_{0}^{3} w_{2}^{2}+2 \mathrm{i} a_{0}^{2} a_{2} w_{2}+a_{0} b_{2}=0,
$$

with respect to functions $h(\theta), w_{2}(\theta)$ and parameters $a_{0}, a_{2}, b_{2}, c_{3}$. If we want to get real potential

$$
V=f_{1}(\theta) \cos (\phi)+f_{2}(\theta)
$$

in (3.7), we have to add one more equation to (3.8)

$$
\mathrm{i} \alpha^{2}\left(a_{0}^{3} w_{2}^{3}-\mathrm{i} a_{0}^{2} a_{2} w_{2}^{2}-a_{0} b_{2} w_{2}+\mathrm{i} c_{3}\right) w_{1}^{2}+a^{4}=0
$$

depending in addition on function $w_{1}$ and parameters $a(3.5)$ and $\alpha$ (3.2).

Some particular solutions of these equation have been studied in [19, 26, 24] including integrable systems due to Goryachev, Chaplygin, Dullin, Matveev etc. For all these systems, we collect $a_{0}$ and the zero-valued coefficients in (3.8) in the following table

\begin{tabular}{|l|l|l|}
\hline Goryachev-Chaplygin top & $a_{0}=2 \mathrm{i} a$ & $b_{2}=c_{3}=0$ \\
Goryachev system & $a_{0}=2 \mathrm{i} a / 3$ & $a_{2}=b_{2}=0$ \\
Case 3 from [19] & $a_{0}=\mathrm{i} a / 3$ & $a_{2}=b_{2}=0$ \\
Dullin-Matveev system & $a_{0}=\mathrm{i} a$ & $c_{3}=0$ \\
Case 5 from [19] & $a_{0}=\mathrm{i} a / 2$ & $a_{2}=c_{3}=0$ \\
\hline
\end{tabular}

Integrable systems with the same coefficients in the separated relations (3.6) and with different $a_{0}$ and $a_{0}^{\prime}$ in (3.5) are related by non-canonical transformation of the momenta

$$
z=a_{0} p_{k} \rightarrow z=a_{0}^{\prime} p_{k}, \quad k=1,2
$$

\subsection{Goryachev-Chaplygin top}

Let us consider Gorychev-Chaplygin top with the following integrals of motion

$$
H_{1}=J_{1}^{2}+J_{2}^{2}+4 J_{3}^{2}+a x_{1}+\frac{b}{x_{3}^{2}}, \quad H_{2}=2 J_{3}\left(J_{1}^{2}+J_{2}^{2}+\frac{b}{x_{3}^{2}}\right)+a x_{3} J_{1}
$$

In this case variables of separation (3.13.3) are determined by

$$
q_{1}+q_{2}=-\frac{2 J_{3}}{x_{3}^{2}}-\frac{J_{1}+\mathrm{i} J_{2}}{x_{3}\left(x_{1}+\mathrm{i} x_{2}\right)}, \quad q_{1} q_{2}=\frac{a}{2 x_{3}^{2}\left(x_{1}+\mathrm{i} x_{2}\right)}, \quad p_{1,2}=\frac{\mathrm{i} x_{3}^{2}}{2}+\frac{\mathrm{i} J_{3}}{q_{1,2}} .
$$


They are related with initial variables by the rule

$$
\begin{aligned}
x_{1}+\mathrm{i} x_{2} & =-\frac{\mathrm{i} a\left(q_{1}-q_{2}\right)}{4 q_{1} q_{1}\left(p_{1} q_{1}-q_{2} p_{2}\right)}, \quad x_{3}=\sqrt{-\frac{2 \mathrm{i}\left(p_{1} q_{1}-q_{2} p_{2}\right)}{q_{1}-q_{2}}} \\
J_{1}+\mathrm{i} J_{2} & =\frac{a\left(q_{1}^{2} p_{1}-q_{2}^{2} p_{2}\right)}{2 q_{1} q_{2}\left(p_{1} q_{1}-q_{2} p_{2}\right) \sqrt{-\frac{2 \mathrm{i}\left(p_{1} q_{1}-q_{2} p_{2}\right)}{q_{1}-q_{2}}}}, \quad J_{3}=\frac{\mathrm{i} q_{1} q_{2}\left(p_{1}-p_{2}\right)}{q_{1}-q_{2}}, \\
x_{1}-\mathrm{i} x_{2} & =\frac{4 q_{1} q_{2}}{a\left(q_{1}-q_{2}\right)^{2}},\left(\left(\mathrm{i}-2 p_{1}\right) q_{1}^{2} p_{1}+\left(4 p_{1} p_{2}-\mathrm{i} p_{1}-\mathrm{i} p_{2}\right) q_{1} q_{2}+\left(\mathrm{i}-2 p_{2}\right) q_{2}^{2} p_{2}\right) \\
J_{1}-\mathrm{i} J_{2} & =-\frac{8 \mathrm{i} q_{1} q_{2}}{a\left(q_{1}-q_{2}\right)^{2} \sqrt{-\frac{2 \mathrm{i}\left(p_{1} q_{1}-q_{2} p_{2}\right)}{q_{1}-q_{2}}}\left(q_{1} p_{1}-q_{2} p_{2}\right)\left(\mathrm{i}-2 p_{1}\right) p_{1} q_{1}^{2}+} \\
+ & \left.\left(q_{1} p_{1}+q_{2} p_{2}\right)\left(\mathrm{i}-2 p_{2}\right) p_{2} q_{2}^{2}\right) .
\end{aligned}
$$

Separated relation is given by equation with real coefficients

$$
\Phi(q, \mu)=\left(\mu^{2}-b\right) q^{2}+\left(\mu^{3}-H_{1} \mu+H_{2}\right) q+\frac{a^{2}}{4}=0, \quad q=q_{1,2}, \quad \mu=2 \mathrm{i} q_{1,2} p_{1,2} .
$$

Equations of motion in variables of separation look like

$$
\begin{aligned}
& \frac{\dot{q}_{1}}{q_{1}\left(3 \mu_{1}^{2}+2 q_{1} \mu_{1}-H_{1}\right)}+\frac{\dot{q}_{2}}{q_{2}\left(3 \mu_{2}^{2}+2 q_{2} \mu_{2}-H_{1}\right)}=0 \\
& \frac{\mu_{1} q_{1}}{q_{1}\left(3 \mu_{1}^{2}+2 q_{1} \mu_{1}-H_{1}\right)}+\frac{\mu_{2} q_{2}}{q_{2}\left(3 \mu_{2}^{2}+2 q_{2} \mu_{2}-H_{1}\right)}=2 \mathrm{i} .
\end{aligned}
$$

By making the birational change

$$
q=\frac{a^{2}}{4 x}, \quad \mu=\frac{z}{x}
$$

the curve (3.12) can be transformed to the canonical trigonal form (3.6) at

$$
a_{1}=b_{1}=b_{2}=c_{2}=c_{3}=0
$$

whereas other parameters are functions on $a, b$.

\subsubsection{Deformation of the Goryachev-Chaplygin top}

Substituting $q=q_{1,2}$ and $\mu=2 \mathrm{i} q_{1,2} p_{1,2}$ into the non-hyperelliptic algebraic curve of genus three defined by the following equation

$$
\Phi_{1}(q, \mu)=c q^{3}+\left(\mu^{2}+d \mu-b\right) q^{2}+\left(\mu^{3}+e \mu^{2}-\widehat{H}_{1} \mu+\widehat{H}_{2}\right) q+\frac{a^{2}}{4}=0
$$

and solving a pair of the resulting equations with respect to $\widehat{H}_{1,2}$ one gets deformation of the initial Hamilton function

$$
\widehat{H}_{1}=J_{1}^{2}+J_{2}^{2}+4 J_{3}^{2}+a x_{1}+\frac{b}{x_{3}^{2}}-\left(e-\frac{c-d+e}{x_{1}^{2}+x_{2}^{2}}+\frac{c}{x_{3}^{2}}-\frac{2 c}{x_{3}^{4}}\right) J_{3}+\frac{\left(c-d x_{3}^{2}+e x_{3}^{4}\right)^{2}}{4 x_{3}^{6}\left(x_{1}^{2}+x_{2}^{2}\right)},
$$

using the generalized shift (1.8) at

$$
f=-\frac{\mathrm{i}\left(e x_{3}^{4}-d x_{3}^{2}+c\right)}{2 \sqrt{1-x_{3}^{2}} x_{3}^{3}}
$$


In this case quadratures are defined by the following differential equations

$$
\begin{aligned}
& \sum_{k=1}^{2} \frac{\dot{q}_{k}}{q_{k}\left(3 \mu_{k}^{2}+2 \mu_{k} q_{k}+2 e \mu_{k}+d q_{k}-\widehat{H}_{1}\right)}=0, \quad \mu_{k}=2 \mathrm{i} q_{k} p_{k} \\
& \sum_{k=1}^{2} \frac{\mu_{k} \dot{q}_{k}}{q_{k}\left(3 \mu_{k}^{2}+2 \mu_{k} q_{k}+2 e \mu_{k}+d q_{k}-\widehat{H}_{1}\right)}=2 \mathrm{i} .
\end{aligned}
$$

If $c=0$ and $d=e$, one gets the usual Goryachev-Chaplygin gyrostat with the Hamiltonian

$$
\widehat{H}_{1}=J_{1}^{2}+J_{2}^{2}+4 J_{3}^{2}-e J_{3}+a x_{1}+\frac{b}{x_{3}^{2}} .
$$

In this case equation (3.14) defines genus two hyperelliptic curve instead of trigonal one.

\subsection{Goryachev system}

Let us consider Gorychev system on the sphere defined by the following integrals of motion

$$
\begin{aligned}
& H_{1}=J_{1}^{2}+J_{2}^{2}+\frac{4}{3} J_{3}^{2}+\frac{a x_{1}}{x_{3}^{2 / 3}}+\frac{b}{x_{3}^{2 / 3}}, \\
& H_{2}=-\frac{2 J_{3}}{3}\left(J_{1}^{2}+J_{2}^{2}+\frac{8}{9} J_{3}^{2}+\frac{b}{x_{3}^{2 / 3}}\right)+\frac{a\left(3 x_{3} J_{1}-2 x_{1} J_{3}\right)}{3 x_{3}^{2 / 3}} .
\end{aligned}
$$

The corresponding variables of separation $q_{1,2}$ and $p_{1,2}$ (3.13.3) are obtained from

$$
q_{1}+q_{2}=\frac{x_{3}^{4 / 3} J_{3}}{1-x_{3}^{2}}+\frac{\mathrm{i}\left(J_{1} x_{2}-x_{1} J_{2}\right) x_{3}^{1 / 3}}{1-x_{3}^{2}}, \quad q_{1} q_{2}=\frac{a}{2\left(x_{1}+\mathrm{i} x_{2}\right)}, \quad p_{1,2}=\frac{3 \mathrm{i} x_{3}^{2 / 3}}{2}+\frac{\mathrm{i} J_{3}}{q_{1,2}} .
$$

Inverse transformation looks like

$$
\begin{aligned}
x_{1}+\mathrm{i} x_{2} & =\frac{a}{2 q_{1} q_{2}}, \quad J_{3}=\frac{\mathrm{i} q_{1} q_{2}\left(p_{1}-p_{2}\right)}{q_{1}-q_{2}}, \\
x_{1}-\mathrm{i} x_{2} & =2 \frac{q_{1} q_{2}\left(1-x_{3}^{2}\right)}{a}, \quad J_{1}+\mathrm{i} J_{2}=-\frac{a\left(q_{1}+q_{2}\right)}{2 q_{1} q_{2} x_{3}^{1 / 3}}, \\
J_{1}-\mathrm{i} J_{2} & =-\frac{4 \mathrm{i} q_{1}^{2} q_{2}^{2}\left(p_{1}-p_{2}\right)}{a\left(q_{1}-q_{2}\right)} x_{3}+\frac{2 q_{1} q_{2}\left(1-x_{3}^{2}\right)\left(q_{1}+q_{2}\right)}{a x_{3}^{1 / 3}},
\end{aligned}
$$

where

$$
x_{3}=\left(-\frac{2 \mathrm{i}\left(p_{1} q_{1}-p_{2} q_{2}\right)}{3\left(q_{1}-q_{2}\right)}\right)^{3 / 2} .
$$

Separated relation is given by equation with real coefficients

$$
\Phi(q, \mu)=q^{4}-b q^{2}+\left(\mu^{3}-H_{1} \mu+H_{2}\right) q+\frac{a^{2}}{4}=0, \quad \text { at } \quad q=q_{1,2}, \quad \mu=\frac{2 \mathrm{i}}{3} q_{1,2} p_{1,2} .
$$

In this case quadratures read as

$$
\begin{aligned}
& \int_{q_{0}}^{q_{1}} \frac{d q}{q\left(3 \mu^{2}-H_{1}\right)}+\int_{q_{0}}^{q_{2}} \frac{d q}{q\left(3 \mu^{2}-H_{1}\right)}=\beta_{1}, \\
& \int_{q_{0}}^{q_{1}} \frac{\mu d q}{q\left(3 \mu^{2}-H_{1}\right)}+\int_{q_{0}}^{q_{2}} \frac{\mu d q}{q\left(3 \mu^{2}-H_{1}\right)}=\frac{2 \mathrm{i}}{3} t+\beta_{2} .
\end{aligned}
$$

As usual, here $\mu$ is a function on $q$ obtained from the separated relation (3.17). 


\subsubsection{Deformation of the Goryachev system}

Using trigonal curve of genus three defined by the following equation

$$
\Phi_{1}=q^{4}+c q^{3}-b q^{2}+\left(\mu^{3}+d \mu^{2}-\widehat{H}_{1} \mu+\widehat{H}_{2}\right) q+\frac{a^{2}}{4}=0,
$$

instead of (3.17) one gets deformation of the initial Hamilton function (3.15)

$$
\widehat{H}_{1}=H_{1}-\left(\frac{d}{3}+\frac{d+c x_{3}^{2 / 3}}{x_{1}^{2}+x_{2}^{2}}\right) J_{3}+\frac{\left(c+d x_{3}^{4 / 3}\right)^{2}}{4\left(x_{1}^{2}+x_{2}^{2}\right) x_{3}^{2 / 3}}
$$

after the generalized shift (1.8) at

$$
f=-\frac{\mathrm{i}\left(c+d x_{3}^{4 / 3}\right)}{2 \sqrt{1-x_{3}^{2}} x_{3}^{1 / 3}} .
$$

The corresponding equations of motion look like

$$
\begin{aligned}
& \frac{\dot{q}_{1}}{q_{1}\left(3 \mu_{1}^{2}+2 d \mu_{1}-\widehat{H}_{1}\right)}+\frac{\dot{q}_{2}}{q_{2}\left(3 \mu_{2}^{2}+2 d \mu_{2}-\widehat{H}_{1}\right)}=0, \quad \mu_{k}=\frac{2 \mathrm{i}}{3} q_{k} p_{k} \\
& \frac{\mu_{1} \dot{q}_{1}}{q_{1}\left(3 \mu_{1}^{2}+2 d \mu_{1}-\widehat{H}_{1}\right)}+\frac{\mu_{1} \dot{q}_{2}}{q_{2}\left(3 \mu_{2}^{2}+2 d \mu_{2}-\widehat{H}_{1}\right)}=\frac{2 \mathrm{i}}{3} .
\end{aligned}
$$

Birational transformation (3.13) maps the curve (3.19) to the canonical trigonal form (3.6) at $a_{2}=$ $b_{1}=b_{2}=0$.

\subsection{Case 3 from [19]}

Let us consider one more integrable system from [19] defined by the following integrals of motion

$$
\begin{aligned}
& H_{1}=J_{1}^{2}+J_{2}^{2}+\left(\frac{1}{12}+\frac{\left(2 x_{3}+1\right)}{2\left(x_{3}+1\right)}\right) J_{3}^{2}+\frac{a x_{1}}{\left(x_{3}+1\right)^{5 / 6}}+\frac{b}{\left(x_{3}+1\right)^{1 / 3}}, \\
& H_{2}=\frac{1}{27} J_{3}^{3}-\frac{1}{3} J_{3} H_{1}-a\left(x_{3}+1\right)^{1 / 6} J_{1}+\frac{a x_{1} J_{3}}{2\left(x_{3}+1\right)^{5 / 6}} .
\end{aligned}
$$

The corresponding variables of separation $q_{1,2}$ and $p_{1,2}$ (3.13.3) are obtained from

$q_{1}+q_{2}=-\frac{\left(1+x_{3}\right)^{2 / 3} J_{3}}{2\left(x_{3}-1\right)}-\frac{\mathrm{i}\left(x_{2} J_{1}-x_{1} J_{2}\right)}{\left(1+x_{3}\right)^{1 / 3}\left(x_{3}-1\right)}, \quad q_{1} q_{2}=\frac{a \sqrt{1+x_{3}}}{2\left(x_{1}+\mathrm{i} x_{2}\right)}, \quad p_{1,2}=3 \mathrm{i}\left(1+x_{3}\right)^{1 / 3}+\frac{\mathrm{i} J_{3}}{q_{1,2}}$.

Inverse transformation looks like

$$
\begin{aligned}
x_{1}+\mathrm{i} x_{2} & =\frac{a \sqrt{1+x_{3}}}{2 q_{1} q_{2}}, \quad J_{3}=\frac{\mathrm{i} q_{1} q_{2}\left(p_{1}-p_{2}\right)}{q_{1}-q_{2}} \\
x_{1}-\mathrm{i} x_{2} & =-\frac{2 q_{1} q_{2}\left(x_{3}^{2}-1\right)}{a \sqrt{1+x_{3}}}, \quad J_{1}+\mathrm{i} J_{2}=\frac{\mathrm{i} a\left(p_{1}-p_{2}\right)}{4\left(q_{1}-q_{2}\right) \sqrt{1+x_{3}}}-\frac{a\left(q_{1}+q_{2}\right)}{2 q_{1} q_{2}\left(1+x_{3}\right)^{1 / 6}}, \\
J_{1}-\mathrm{i} J_{2} & =-\frac{\mathrm{i} q_{1}^{2} q_{2}^{2}\left(3 x_{3}+1\right)\left(p_{1}-p_{2}\right)}{a\left(q_{1}-q_{2}\right) \sqrt{1+x_{3}}}-\frac{2 q_{1} q_{2}\left(q_{1}+q_{2}\right)\left(x_{3}-1\right)}{a\left(1+x_{3}\right)^{1 / 6}}
\end{aligned}
$$

where

$$
x_{3}=\frac{\mathrm{i}\left(p_{1} q_{1}-p_{2} q_{2}\right)^{3}}{27\left(q_{1}-q_{2}\right)^{3}}-1 .
$$

Separated relations are defined by equation with the real coefficients

$$
\Phi(q, \mu)=2 q^{4}-b q^{2}+\left(\mu^{3} q-H_{1} \mu+H_{2}\right) q+\frac{a^{2}}{4}=0, \quad q=q_{1,2}, \quad \mu=\frac{\mathrm{i} q_{1,2} p_{1,2}}{3}
$$


The corresponding quadratures are given by

$$
\begin{aligned}
& \int_{q_{0}}^{q_{1}} \frac{\dot{q}}{q\left(3 \mu^{2}-H_{1}\right)}+\int_{q_{0}}^{q_{2}} \frac{\dot{q}}{q\left(3 \mu^{2}-H_{1}\right)}=\beta_{1}, \\
& \int_{q_{0}}^{q_{1}} \frac{\mu \dot{q}}{q\left(3 \mu^{2}-H_{1}\right)}+\int_{q_{0}}^{q_{2}} \frac{\mu \dot{q}}{q\left(3 \mu^{2}-H_{1}\right)}=\frac{\mathrm{i}}{3} t+\beta_{2} .
\end{aligned}
$$

As for the Goryachev system, birational change (3.13) transforms the equation (3.23) to the canonical trigonal form (3.6) at

$$
a_{1}=a_{2}=b_{1}=b_{2}=c_{2}=0,
$$

It allows us to prove that integrals of motion for this system (3.21) are related with integrals of motion (3.15) for the Goryachev system by the non-canonical transformation (3.10).

It may seem that quadratures (3.18) and (3.24) are trivially related by change of time

$$
t \rightarrow 2 t
$$

but we have to keep firmly in mind that $\mu$ in (3.18) is a function on $q$ obtained from (3.17), whereas $\mu$ in (3.24) is another function on $q$ obtained from (3.23).

\subsubsection{Deformation of the system (3.21)}

Similar to the Goryachev system, we can add two term to the initial trigonal curve of genus three (3.23)

$$
\Phi_{1}=2 q^{4}+c q^{3}-b q^{2}+\left(\mu^{3}+d \mu^{2}-\widehat{H}_{1} \mu+\widehat{H}_{2}\right) q+\frac{a^{2}}{4}=0 .
$$

Deformation of the initial Hamilton function (3.21) looks like

$$
\widehat{H}_{1}=H_{1}-\left(\frac{d}{6}-\frac{d}{x_{3}-1}-\frac{c\left(1+x_{3}\right)^{1 / 3}}{2\left(x_{3}-1\right)}\right) J_{3}+\frac{\left(d \sqrt{1+x_{3}}+c\left(1+x_{3}\right)^{-1 / 6}\right)^{2}}{4\left(1-x_{3}\right)}
$$

after canonical transformation (1.8) at

$$
f=-\frac{\mathrm{i}\left(d\left(1+x_{3}\right)+c\left(1+x_{3}\right)^{1 / 3}\right)}{2 \sqrt{1-x_{3}^{2}}} .
$$

In this case equations of motion are equal to

$$
\begin{aligned}
& \frac{\dot{q}_{1}}{q_{1}\left(3 \mu_{1}^{2}+2 d \mu_{1}-\widehat{H}_{1}\right)}+\frac{\dot{q}_{2}}{q_{2}\left(3 \mu_{2}^{2}+2 d \mu_{2}-\widehat{H}_{1}\right)}=0, \quad \mu_{k}=\frac{\mathrm{i}}{3} q_{k} p_{k}, \\
& \frac{\mu_{1} \dot{q}_{1}}{q_{1}\left(3 \mu_{1}^{2}+2 d \mu_{1}-\widehat{H}_{1}\right)}+\frac{\mu_{2} \dot{q}_{2}}{q_{2}\left(3 \mu_{2}^{2}+2 d \mu_{2}-\widehat{H}_{1}\right)}=\frac{\mathrm{i}}{3} .
\end{aligned}
$$

\subsection{Dullin-Matveev system}

Let us consider the Dullin-Matveev system [5] defined by the following integrals of motion

$$
\begin{aligned}
H_{1} & =J_{1}^{2}+J_{2}^{2}+\left(1+\frac{x_{3}}{x_{3}+c}-\frac{x_{3}^{2}-|x|^{2}}{4\left(x_{3}+c\right)^{2}}\right) J_{3}^{2}+\frac{a x_{1}}{\left(x_{3}+c\right)^{1 / 2}}+\frac{b}{x_{3}+c} \\
H_{2} & =-\left(J_{1}^{2}+J_{2}^{2}-\frac{J_{3}^{2}}{4}+\frac{\left(4 x_{3}^{2}+6 x_{3} c+c^{2}+|x|^{2}\right) J_{3}^{2}}{4\left(x_{3}+c\right)^{2}}+\frac{b}{x_{3}+c}\right) J_{3} \\
& +a \sqrt{x_{3}+c} J_{1}-\frac{a x_{1} J_{3}}{2 \sqrt{x_{3}+c}} .
\end{aligned}
$$


According to [24] variables of separation $q_{1,2}$ and $p_{1,2}$ are defined by (3.1/3.3)

$$
q_{1}+q_{2}=-\frac{J_{3}}{2\left(c+x_{3}\right)}-\frac{J_{1}+\mathrm{i} J_{2}}{x_{1}+\mathrm{i} x_{2}}, \quad q_{1} q_{2}=\frac{a}{2\left(x_{1}+\mathrm{i} x_{2}\right) \sqrt{c+x_{3}}}, \quad p_{1,2}=\mathrm{i}\left(c+x_{3}\right)+\frac{\mathrm{i} J_{3}}{q_{1,2}}
$$

or by inverse transformation

$$
\begin{aligned}
x_{1}+\mathrm{i} x_{2} & =\frac{a}{2 \sqrt{-\frac{\mathrm{i}\left(p_{1} q_{1}-p_{2} q_{2}\right)}{q_{1}-q_{2}}} q_{1} q_{2}}, \quad x_{3}=-\frac{\mathrm{i}\left(p_{1} q_{1}-q_{2} p_{2}\right)}{q_{1}-q_{2}}-c, \quad J_{3}=\frac{\mathrm{i} q_{1} q_{2}\left(p_{1}-p_{2}\right)}{q_{1}-q_{2}}, \\
x_{1}-\mathrm{i} x_{2} & =-\frac{2 \sqrt{-\frac{\mathrm{i}\left(p_{1} q_{1}-p_{2} q_{2}\right)}{q_{1}-q_{2}}} q_{1} q_{2}}{a\left(q_{1}-q_{2}\right)^{2}}\left(\left(c+1+\mathrm{i} p_{1}\right) q_{1}-\left(c+1+\mathrm{i} p_{2}\right) q_{2}\right) \\
& \times\left(\left(c-1+\mathrm{i} p_{1}\right) q_{1}-\left(c-1+\mathrm{i} p_{2}\right) q_{2}\right), \\
J_{1}+\mathrm{i} J_{2} & =-\frac{a\left(q_{1}\left(2 q_{1}+q_{2}\right) p_{1}-q_{2}\left(2 q_{2}+q_{1}\right) p_{2}\right)}{4 \sqrt{-\frac{\mathrm{i}\left(p_{1} q_{1}-p_{2} q_{2}\right)}{q_{1}-q_{2}}}, q_{1} q_{2}\left(p_{1} q_{1}-p_{2} q_{2}\right)}, \\
J_{1}-\mathrm{i} J_{2} & =\frac{\mathrm{i} q_{1} q_{2}}{a \sqrt{-\frac{\mathrm{i}\left(p_{1} q_{1}-p_{2} q_{2}\right)}{q_{1}-q_{2}}}\left(q_{1}-q_{2}\right)}\left(\left(c+1+\mathrm{i} p_{1}\right)\left(c-1+\mathrm{i} p_{1}\right)\left(2 p_{1} q_{1}-3 q_{2} p_{1}-p_{2} q_{2}\right) q_{1}^{3}\right. \\
& +\left(2 \mathrm{i}\left(p_{1}+p_{2}\right) c-4 p_{1} p_{2}\right)\left(p_{1}-p_{2}\right) q_{1}^{2} q_{2}^{2}-\left(c+1+\mathrm{i} p_{2}\right)\left(c-1+\mathrm{i} p_{2}\right)\left(2 p_{2} q_{2}-3 q_{1} p_{2}-p_{1} q_{1}\right) q_{2}^{3} .
\end{aligned}
$$

The corresponding separated relations are defined by equation with the real coefficients

$$
\Phi(q, \mu)=\mu\left(c^{2}-1\right) q^{3}+\left(2 c \mu^{2}-b\right) q^{2}+\left(\mu^{3}-H_{1} \mu+H_{2}\right) q+\frac{a^{2}}{4}=0, \quad q=q_{1,2}, \quad \mu=\mathrm{i} q_{1,2} p_{1,2},
$$

and quadratures in differential form look like

$$
\begin{aligned}
& \frac{\dot{q_{1}}}{q_{1}\left(\left(c^{2}-1\right) q_{1}^{2}+4 c q_{1} \mu_{1}+3 \mu_{1}^{2}-H_{1}\right)}+\frac{\dot{q_{2}}}{q_{2}\left(\left(c^{2}-1\right) q_{2}^{2}+4 c q_{2} \mu_{2}+3 \mu_{2}^{2}-H_{1}\right)}=0, \\
& \frac{\mu_{1} \dot{q_{1}}}{q_{1}\left(\left(c^{2}-1\right) q_{1}^{2}+4 c q_{1} \mu_{1}+3 \mu_{1}^{2}-H_{1}\right)}+\frac{\mu_{2} \dot{q_{2}}}{q_{2}\left(\left(c^{2}-1\right) q_{2}^{2}+4 c q_{2} \mu_{2}+3 \mu_{2}^{2}-H_{1}\right)}=\mathrm{i} .
\end{aligned}
$$

\subsubsection{Deformation of the Dullin-Matveev system}

Substituting $q=q_{1,2}$ and $\mu=\mathrm{i} q_{1,2} p_{1,2}$ into the non-hyperelliptic algebraic curve of genus three defined by the following equation

$$
\Phi_{1}=\mu\left(c^{2}-1\right) q^{3}+\left(2 c \mu^{2}+d \mu-b\right) q^{2}+\left(\mu^{3}+e \mu^{2}-\widehat{H}_{1} \mu+\widehat{H}_{2}\right) q+\frac{a^{2}}{4}=0,
$$

and solving a pair of the resulting equations with respect to $H_{1,2}$ one gets deformation of the initial Hamilton function (3.28)

$$
\widehat{H}_{1}=H_{1}-\frac{1}{2}\left(e-\frac{d}{c+x_{3}}+\frac{(c e-d) x_{3}+e}{x_{1}^{2}+x_{2}^{2}}\right) J_{3}+-\frac{\left(c e-d+x_{3} e\right)^{2}}{4\left(x_{1}^{2}+x_{2}^{2}\right)}
$$

after the generalized shift (1.8) at

$$
f=-\frac{\mathrm{i}\left(c e-d+x_{3} e\right)}{2 \sqrt{1-x_{3}^{2}}} .
$$


Using the same birational change (3.13) the curve (3.19) can be transformed to the canonical trigonal form (3.6) at $c_{2}=c_{3}=0$.

In this case equations of motion read as

$$
\begin{aligned}
& \sum_{k=1}^{2} \frac{\dot{q}_{k}}{q_{k}\left(3 \mu_{k}^{2}+4 c q_{k} \mu_{k}+2 e \mu_{k}+q_{k}^{2}\left(c^{2}-1\right)+d q_{k}-\widehat{H}_{1}\right)}=0, \quad \mu_{k}=\mathrm{i} q_{k} p_{k}, \\
& \sum_{k=1}^{2} \frac{\mu_{k} \dot{q}_{k}}{q_{k}\left(3 \mu_{k}^{2}+4 c q_{k} \mu_{k}+2 e \mu_{k}+q_{k}^{2}\left(c^{2}-1\right)+d q_{k}-\widehat{H}_{1}\right)}=\mathrm{i}
\end{aligned}
$$

\subsection{Case 5 from 19}

Let us consider last integrable system from [19] with integrals of motion

$$
\begin{aligned}
& H_{1}=J_{1}^{2}+J_{2}^{2}+\left(\frac{3}{16}+\frac{8 x_{3}+5}{8\left(x_{3}+1\right)}\right) J_{3}^{2}+\frac{a x_{1}}{\left(x_{3}+1\right)^{3 / 4}}+\frac{b}{\sqrt{x_{3}+1}}, \\
& H_{2}=\frac{1}{8} J_{3}^{3}-\frac{1}{2} H_{1} J_{3}+a\left(x_{3}+1\right)^{1 / 4} J_{1}--\frac{a x_{1} J_{3}}{4\left(x_{3}+1\right)^{3 / 4}} .
\end{aligned}
$$

The corresponding variables of separation $q_{1,2}$ and $p_{1,2}$ (3.113.3) are obtained from

$q_{1}+q_{2}=\frac{\left(3 x_{3}+1\right) J_{3}}{4 \sqrt{x_{3}+1}\left(1-x_{3}\right)}+\frac{\mathrm{i}\left(x_{2} J_{1}-x_{1} J_{2}\right)}{\sqrt{x_{3}+1}\left(1-x_{3}\right)}, \quad q_{1} q_{2}=\frac{a\left(x_{3}+1\right)^{1 / 4}}{2\left(x_{1}+\mathrm{i} x_{2}\right)}, \quad p_{1,2}=2 \mathrm{i} \sqrt{x_{3}+1}+\frac{\mathrm{i} J_{3}}{q_{1,2}}$.

Inverse transformation looks like

$$
\begin{aligned}
x_{1}+\mathrm{i} x_{2} & =\frac{a\left(x_{3}+1\right)^{1 / 4}}{2 q_{1} q_{2}}, \quad J_{3}=\frac{\mathrm{i} q_{1} q_{2}\left(p_{1}-p_{2}\right)}{q_{1}-q_{2}}, \\
x_{1}-\mathrm{i} x_{2} & =-\frac{2 q_{1} q_{2}\left(x_{3}^{2}-1\right)}{a\left(x_{3}+1\right)^{1 / 4}}, \quad J_{1}+\mathrm{i} J_{2}=\frac{\mathrm{i} a\left(p_{1}-p_{2}\right)}{8\left(q_{1}-q_{2}\right)\left(x_{3}+1\right)^{3 / 4}}-\frac{a\left(q_{1}+q_{2}\right)}{2 q_{1} q_{2}\left(x_{3}+1\right)^{1 / 4}}, \\
J_{1}-\mathrm{i} J_{2} & =-\frac{\mathrm{i} q_{1}^{2} q_{2}^{2}\left(7 x_{3}+1\right)\left(p_{1}-p_{2}\right)}{2 a\left(q_{1}-q_{2}\left(\left(x_{3}+1\right)^{1 / 4}\right.\right.}-\frac{2 q_{1} q_{2}\left(q_{1}+q_{2}\right)\left(x_{3}-1\right)\left(x_{3}+1\right)^{1 / 4}}{a},
\end{aligned}
$$

where

$$
x_{3}=-\frac{\left(p_{1} q_{1}-p_{2} q_{2}\right)^{2}}{4\left(q_{1}-q_{2}\right)^{2}}-1 .
$$

Separated relations are defined by

$$
\Phi(q, \mu)=-2 \mu q^{3}-b q^{2}+\left(\mu^{3}-H_{1} \mu+H_{2}\right) q+\frac{a^{2}}{4}=0, \quad q=q_{1,2}, \quad \mu=\frac{\mathrm{i}}{2} q_{1,2} p_{1,2}
$$

and we have the following quadratures in differential form

$$
\begin{aligned}
& \frac{\dot{q}_{1}}{q_{1}\left(3 \mu_{1}^{2}-H_{1}-2 q_{1}^{2}\right)}+\frac{\dot{q}_{2}}{q_{2}\left(3 \mu_{2}^{2}-H_{1}-2 q_{2}^{2}\right)}=0, \\
& \frac{\mu_{1} \dot{q}_{1}}{q_{1}\left(3 \mu_{1}^{2}-H_{1}-2 q_{1}^{2}\right)}+\frac{\mu_{2} \dot{q}_{2}}{q_{2}\left(3 \mu_{2}^{2}-H_{1}-2 q_{2}^{2}\right)}=\frac{\mathrm{i}}{2} .
\end{aligned}
$$




\subsubsection{Deformation of the system (3.34)}

Let us add three terms to initial trigonal curve of genus three (3.36)

$$
\Phi_{1}=(c-2 \mu) q^{3}-(d \mu+b) q^{2}+\left(\mu^{3}+e \mu^{2}-\widehat{H}_{1} \mu+\widehat{H}_{2}\right) q+\frac{a^{2}}{4}=0 .
$$

The corresponding deformation of the initial Hamilton function (3.34) has the form

$$
\begin{aligned}
\widehat{H}_{1} & =H_{1}-\left(\frac{e}{4}+\frac{c+2 e}{2\left(1-x_{3}\right)}+\frac{c}{4\left(1+x_{3}\right)}+\frac{d\left(x_{3}^{2}+4 x_{3}+3\right)}{4\left(1-x_{3}\right)\left(1+x_{3}\right)^{3 / 2}}\right) J_{3} \\
& +\frac{1}{4\left(1-x_{3}\right)}\left(e \sqrt{1+x_{3}}+d+\frac{c}{\sqrt{1+x_{3}}}\right)^{2},
\end{aligned}
$$

after canonical transformation (1.8) at

$$
f=-\frac{\mathrm{i} c+\mathrm{i} e\left(1+x_{3}\right)}{2 \sqrt{1-x_{3}^{2}}}-\frac{\mathrm{i} d}{2 \sqrt{1-x_{3}}} .
$$

The corresponding quadratures are defined by

$$
\begin{aligned}
& \sum_{k=1}^{2} \frac{\dot{q}_{k}}{q_{k}\left(3 \mu_{k}^{2}+2 e \mu_{k}-2 q_{k}^{2}-d q_{k}-\widehat{H}_{1}\right)}=0, \quad \mu_{k}=\frac{\mathrm{i}}{2} q_{k} p_{k} \\
& \sum_{k=1}^{2} \frac{\mu_{k} \dot{q}_{k}}{q_{k}\left(3 \mu_{k}^{2}+2 e \mu_{k}-2 q_{k}^{2}-d q_{k}-\widehat{H}_{1}\right)}=\frac{\mathrm{i}}{2}
\end{aligned}
$$

Non canonical transformations (3.10) relate this equations (3.40) with similar equations (3.33) for the deformed Dullin-Matveev system.

\section{Conclusion}

In 21, 22, 26] some new variables of separation for various integrable systems on the sphere with higher order integrals of motion have been obtained by brute force method. In [23, 24] we introduce a concept of natural Poisson, which allows us to understand the geometric origin of this method and to find some common attributes of the variables of separation for the Kowalevski top, Chaplygin system, Goryachev-Chaplygin gyrostat, Goryachev and Dulllin-Matveev systems etc.

In this more technical paper we continue our investigations in order to explicitly describe canonical transformations of initial physical variables to variables of separation and vice versa, to calculate the corresponding quadratures and to discuss possible integrable deformations of these systems associated with genus three hyperelliptic and non-hyperelliptic algebraic curves.

In Section 2 we consider real variables of separation for which the separation relations have the real coefficients only. In Section 3 we discuss complex variables of separation and the separation relations with the real coefficients as above. Similar complex variables satisfying to the real separated equations for the Kowalevski top and Goryachev-Chaplygin gyrostat have been found in [14, for the Kowalevski-Goryachev-Chaplygin gyrostat in [18] and for the Steklov-Lyapunov system in [25]. These and other known complex variables lying on the real algebraic curves are discussed in the book [2].

Further inquiry is related with numerical, algebro-geometric and topological analysis of the obtained quadratures. For dynamical systems associated with the $(3,4)$ trigonal curve (3.6) we also want to discuss an application of the Kowalevski-Painleve criteria to these systems, because in generic case solutions of the corresponding quadratures are non-meromorphic functions of time.

We would like to thank A.V. Borisov and Yu.N. Fedorov for helpful discussions. 


\section{References}

[1] E.D. Belokolos, A.I. Bobenko, V.Z. Enolskii, A.R. Its, V.B. Matveev, Algebro-geometrical approach to nonlinear integrable equations Springer Series in Nonlinear Dynamics, Berlin: Springer-Verlag 1994, XII+320 p.

[2] A.V. Borisov, I.S. Mamaev, Rigid Body Dynamics. Hamiltonian Methods, Integrability, Chaos, Moscow-Izhevsk, RCD, 2005.

[3] V. M. Buchstaber, V. Z. Enolskii, D. V. Leikin, Kleinian functions, hyperelliptic Jacobians and applications, Amer. Math. Soc. Transl., Ser. 2, Vol. 179, Amer. Math. Soc., Providence, RI, 1997, pp. 133.

[4] S.A. Chaplygin, A new partial solution of the problem of motion of a rigid body in liquid, Trudy Otdel. Fiz. Nauk Obsh. Liub. Est., v.11, no. 2, p.710, 1903.

[5] H.R. Dullin, V.S. Matveev, A new integrable system on the sphere, Mathematical Research Letters, v.11, p.715-722, 2004.

[6] Yu. Fedorov, Classical integrable systems related to generalized Jacobians, Acta Appl. Math., v.55, n. 3, p. 151201, 1999.

[7] D.N. Goryachev, New cases of a rigid body motion about a fixed point, Warshav. Univ. Izv., v.3, p.1-11, 1915.

[8] D.N. Goryachev, New cases of integrability of Eulers dynamical equations, Warsaw Univ. Izv., v.3, p. 113, 1916.

[9] Yu.A. Grigoryev, A.V.Tsiganov, Symbolic software for separation of variables in the HamiltonJacobi equation for the L-systems, Regular and Chaotic Dynamics, v.10(4), p.413-422, 2005.

[10] C.G. Jacobi., Vorlesungen über Dynamik, Königsberg University 1842 - 1843 (edited by Clebsch and published from Reimer, Berlin, 1884)

[11] I.V. Komarov, V.V. Sokolov, A.V. Tsiganov, Poisson maps and integrable deformations of Kowalevski top., J. Phys. A., v.36, p. 8035-8048, 2003.

[12] F. Kotter, Sur le cas traité par Mme Kowalevski de rotation d'un corps solide pesant autor d'un point fixe, Acta Mathematica. v.17, n.1-2. p. 209-263, 1893.

[13] S. Kowalevski, Sur le probléme de la rotation d'un corps solide autour d'un point fixe, Acta Math., 12, 177-232, 1889.

[14] V. B. Kuznetsov Simultaneous separation for the Kowalevski and GoryachevChaplygin gyrostats, J. Phys. A: Math. Gen. v.35, p.6419 , 2002.

[15] J. Liouville, Note sur les equations de la dynamique, J. Math. Pures Appl. v.20, p. 137-138, 1855.

[16] C. Neumann, De problemate quodam mechanico, quod ad primam integralium ultraellipticorum classem revocatur, J. Reine Angew.Math., v.56, p. 46-63, 1859.

[17] P. Stäckel, Über die Integralen der Hamilton-Jacobischen Differential Gleichung mittelst Separation der Variable, Habilitationsschrift, Halle, 1891.

[18] A.V. Tsiganov, On the Kowalevski-Goryachev-Chaplygin gyrostat, J. Phys. A, Math. Gen. v.35, No.26, L309-L318, 2002.

[19] A.V. Tsiganov, On a family of integrable systems on $\mathcal{S}^{2}$ with a cubic integral of motion, J. Phys. A, Math. Gen. v.38, p.921-927, 2005.

[20] A.V. Tsiganov, On the two different bi-Hamiltonian structures for the Toda lattice, J. Phys. A: Math. Theor. v.40, pp. 6395-6406, 2007 
[21] A.V. Tsiganov, On the generalized Chaplygin system, Journal of Mathematical Sciences, v.168, n.8, p.901-911, 2010.

[22] A.V. Tsiganov, New variables of separation for particular case of the Kowalevski top, Regular and Chaotic Dynamics, v.15, n.6, p. 657-667, 2010.

[23] A. V. Tsiganov, On bi-integrable natural Hamiltonian systems on the Riemannian manifolds, arXiv: 1006.3914, accepted to Journal of Nonlinear Mathematical Physics, 2010.

[24] A. V. Tsiganov, On natural Poisson bivectors on the sphere, J. Phys. A: Math. Theor., v.44, 105203 (15pp), 2011.

[25] A. V. Tsiganov, New variables of separation for the Steklov-Lyapunov system, Preprint: arXiv:1101.4345v1, 2011.

[26] A.V. Vershilov, A.V. Tsiganov, On bi-Hamiltonian geometry of some integrable systems on the sphere with cubic integral of motion, J. Phys. A: Math. Theor. v.42, 105203 (12pp), 2009.

[27] A.V. Vershilov, A.V. Tsiganov, On one integrable system with a cubic first integral, Preprint arXiv:1103.1444v $1,2011$.

[28] K. Weierstrass, Mathematische Werke I, vol. 1, 1894.

[29] H.M. Yehia, A.A. Elmandouh, New integrable systems with a quartic integral and new generalizations of Kovalevskayas and Goriatchevs cases, Regular and Chaotic Dynamics, v.13(1), pp. 56 $69,2008$. 